\title{
Specificities of RHIC heavy ion collisions probed by strange particles in STAR experiment.
}

\author{
Christelle Roy for the STAR Collaboration* \\ SUBATECH \\ Nantes, France \\ E-mail: Christelle.Roy@subatech.in2p3.fr
}

RHIC experiments revealed the creation of an atypical nuclear matter at the collider top energy. Among the plethora of new results, some of the most exciting ones were provided by the STAR experiment and in particular in the strangeness sector. Strange particles provide new insights in the global characterization of the chemical and dynamical properties of this so particular new form of matter. They also may help to answer to one of the still open questions which is how quarks hadronize. Some hints can be found from studies related to particle production yields as well as to nuclear modification factors.

HEP2005 International Europhysics Conference on High Energy Physics

21-27 Jul 2005

Lisboa, Portugal

${ }^{*}$ Speaker. 


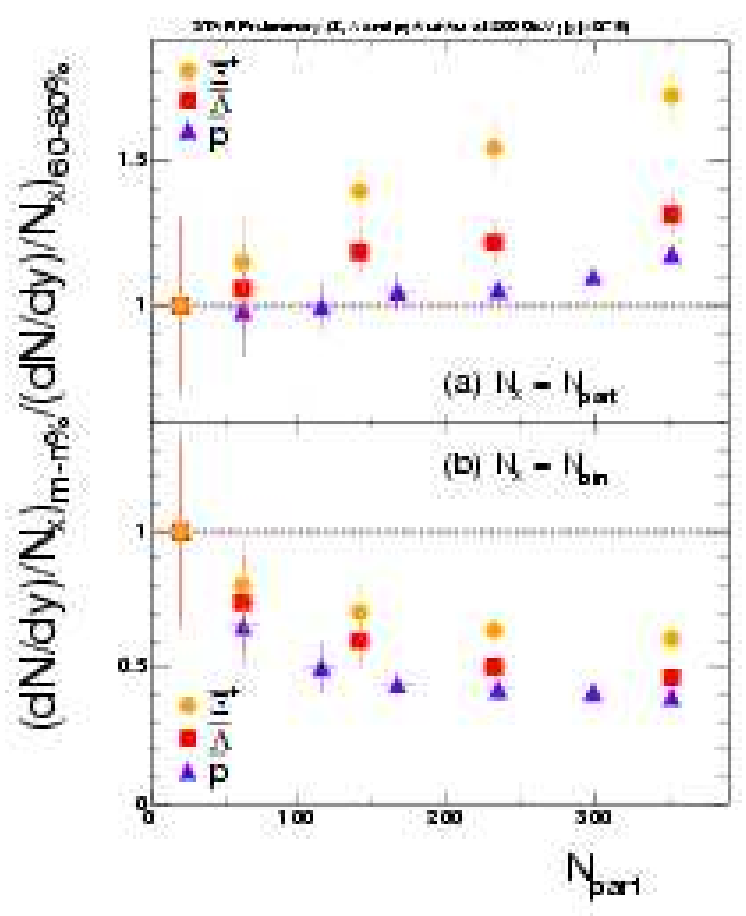

Figure 1: Yields of $\bar{p}, \bar{\Lambda}$ and $\bar{\Xi}$ as a function of centrality (expressed with the number of participants) in $\mathrm{Au}-\mathrm{Au}$ collisions normalized to the most peripheral point and to $N_{\text {part }}$ (upper frame) or $N_{\text {bin }}$ (lower frame).

\section{Foreword}

Since the early 80 's, strange particles are one of the favorite subject of investigation in the search of Quark Gluon Plasma [1]. Since they are created during the collision via gluon-gluon fusion at ultra-relativistic energies, hyperon properties should be marked a priori by the memory of their formation. The STAR experiment involves undoubtedly the most appropriate subsystems for measuring strange particles at RHIC and its results in the strangeness sector have demonstrated the capability of hyperons to provide novel insights in ultra-relativistic heavy ion collisions.

Results discussed here are related to Au-Au collisions at $\sqrt{s_{N N}}=200 \mathrm{GeV}$. p-p collisions at the same energy are used as a reference. The transverse (or mass) momentum spectra of identified particles have been extensively investigated. From their magnitude, chemical properties of the system are extracted while the understanding of their shape points out the dynamical properties.

\section{Understanding hadronization by yield counting}

How hadronization takes place and which processes govern this collision phase are still open and non trivial questions. As far as strangeness is concerned, it is indeed quite puzzling to observe that even if two orders of magnitude separate AGS and RHIC top energies, yields of strange particles do not increase accordingly. Strange antiparticle yields exhibit a more comprehensive behavior showing a strong enhancement in agreement with the decrease of the baryochemical potential. 
To probe the processes governing this production, yields of (anti-)strange particles have been normalized as a function of the number of participants $N_{\text {part }}$ (usually related to soft processes) and of the number of binary collisions $N_{\text {bin }}{ }^{1}$ (supposed to describe hard processes) for Au-Au collisions as it is shown on upper and lower frames of Figure 1, respectively. Strange particles deviate from the $N_{\text {part }}$ scaling and follow better a scaling with $N_{\text {bin }}$. The stranger the particle the stronger the effects. From these observations, a new scaling factor [2] has been proposed assuming that light quarks depend on $N_{\text {part }}$ and strange quarks on $N_{\text {bin }}$. The yields of particles normalized to this new factor scale now for any hadrons. Despite the fact that a physical meaning has to be found for this factor, questions remain open whether it is relevant to describe the centrality of the collision with $N_{\text {part }}$, whether strange quarks and light ones see a different volume of reaction or to go further, whether strange particles are formed like charmed quarks at the early beginning of the reaction.

\section{Understanding hadronization by yield comparison}

Comparison of yields in different ion systems by using nuclear modification factors such as $R_{C P}$ (involving Central and Peripheral collisions) and $R_{A A}$ (involving $\mathrm{Au}-\mathrm{Au}$ and p-p collisions) should provide information on the hadronization. $R_{C P}$ highlighted the particle type dependence at intermediate $p_{T}\left(2 \leq p_{T} \leq 6 \mathrm{GeV}\right)$ as it was suggested by coalescence models [3] leading to the idea that hadrons result from the coalescence of quarks in the dense medium. At higher $p_{T}$, jet fragmentation becomes the dominant process to explain the hadron formation. Hence, the quark constituents may be the relevant degrees of freedom for the description of the collision. This idea emerged also from the studies related to transverse and elliptic flows. Using the blastwave parametrization [4], it has been demonstrated that multi-strange particle transverse momenta can not be reproduced [5] with the same kinetic freeze-out parameters (kinetic temperature and transverse flow velocity) as for light particles. These results suggest that multi-strange baryons decouple earlier from the system in agreement with their supposed low interaction cross-sections and that their non-negligible flow was developed before hadronization occurs, hence at a partonic level. This point was corroborated by elliptic flow studies [6].

Coming back to our comparisons, contrarily to $R_{C P}$ and to mesons, $R_{A A}$ of strange baryons exceed the value of 1 at intermediate $p_{T}$. Here again, the interpretation of the discrepancy between the two nuclear modification factors is not so trivial since one has to understand what differs between p-p and the most peripheral Au-Au collisions, if it has to be understood as a sign of a canonical suppression in p-p collisions or if the suggestion from Topor Pop and collaborators is reasonable or no : Their model Hijing/B $\bar{B}$ v2.0 [7] reproduces the measured $R_{C P}$ as well as the $R_{A A}$ shown in Figure 2 for both mesons and baryons by including Strong Color Field to which strange baryons are more sensitive than the other particles. This effect leads to an enhancement in their production in the most central collisions where the magnitude of the field is the greater.

Whatever the particle production is, each species is created at the chemical freeze-out. It is well-known nowadays that statistical models [8] are very successful in the description of particle ratios by adjusting some thermodynamical parameters. STAR data have been reproduced by adjusting three parameters : a chemical freeze-out temperature similar to that obtained at SPS energy

\footnotetext{
${ }^{1} N_{\text {part }}$ and $N_{\text {bin }}$ are calculated in the framework of the Glauber model.
} 


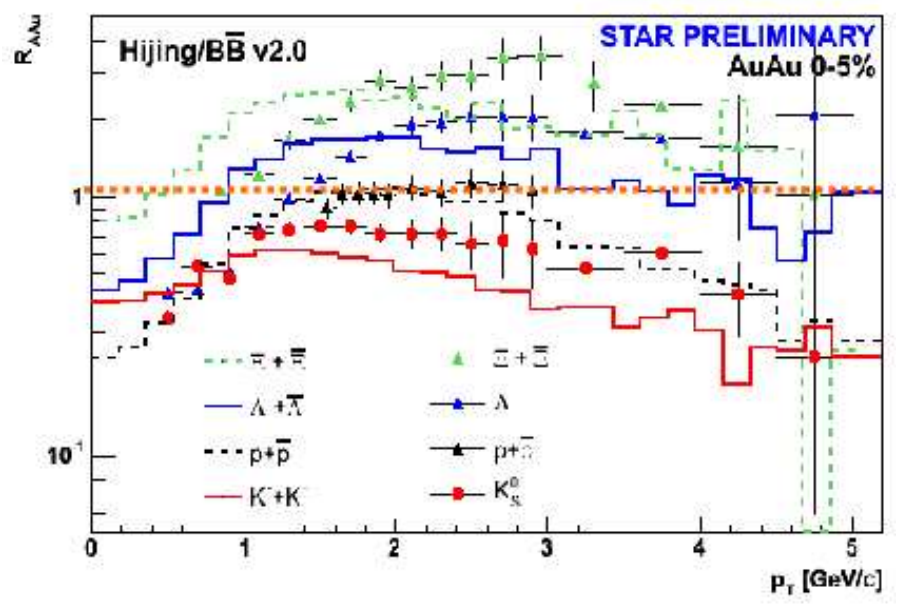

Figure 2: $R_{A A}$ of various hadrons measured by STAR (symbols) and compared to Hijing/B $\bar{B}$ model (histograms).

and to the temperature predicts by QCD for the phase transition, a weak baryonic potential and a strangeness fugacity which reaches unity in the Au-Au most central collisions at RHIC top energy. Strangeness equilibrium seems reached at RHIC.

\section{What we have learned from strangeness}

Strangeness appears to saturate at RHIC and the large amount of produced strange particles allowed numerous precise studies. Looking at specifically strange baryons led quite often to a new interpretation of the previous inclusive measurements. The flow of strange particles combined to their $R_{C P}$ pointed out the emergence of coalescence mechanisms to explain the hadronization at intermediate $p_{T}$. Furthermore, their $R_{A A}$ studies led to still non understood and fundamental points such as the difference between p-p and peripheral heavy ion collisions. The creation time seems different for light quarks and strange ones and even if it may be quite intuitive, it is induced by an unknown underlying process.

\section{References}

[1] For a recent review see : the Strange Quark Matter Proceedings published in J. Phys.G31 (2005).

[2] H. Caines for the STAR Collaboration, J. Phys.G31 (2005) S1057.

[3] D. Molnar et al., Phys. Rev. Lett. 91 (2003) 092301.

[4] E. Schnedermann et al., Phys. Rev. C48 (1993) 2462.

[5] M. Estienne for the STAR Collaboration, Nucl. Phys. A749 (2005) 299.

[6] J. Castillo for the STAR Collaboration, J. Phys. G.: Nucl. Part. Phys 30 (2004) S827.

[7] V. Topor Pop et al., Phys. Rev. C70 (2004) 064906.

[8] M. Kaneta and N. Xu, Nucl. Phys. A698 (2002) 306c; I. Kraus et al., These proceedings. 